Discussion Current practice at our Centre, as judged against the BSPAR/RCO standards of care, is suboptimal. We report a delay in access to an Ophthalmologist for initial slit-lamp examination following JIA diagnosis and follow-up reviews are less frequent than is best practice. This audit has highlighted need for improvement in the provision of care for our patients with JIA and we intend to utilise this data as the basis for future quality improvement initiatives.

\section{G319(P) DRAMATIC RESPONSE OF FAMILIAL MAJEED SYNDROME TO INTERLEUKIN-1 ANTAGONIST THERAPY: CASE REPORT}

'B Almoosawi, ${ }^{2} Z$ Almusawi, ${ }^{2} \mathrm{~W}$ Madan, ${ }^{3} \mathrm{~S}$ Alwadaei, ${ }^{4} \mathrm{H}$ Naser. 'General Paediatrics, RCPCH, Liverpool, UK; ${ }^{2}$ SPaediatric, Salmaniya Medical Complex, Manama, Bahrain; ${ }^{3}$ Neurology, RCP, Birmingham, UK; ${ }^{4}$ Radiology, Salmaniya Medical Complex, Manama, Bahrain

\subsection{6/archdischild-2020-rcpch.275}

Majeed syndrome (MS) is a rare, autosomal recessive, autoinflammatory disease characterized by recurrent multifocal osteomyelitis, congenital dyserythropoietic anemia, and inflammatory dermatome. In this article, we report the cases of two siblings with MS. Genetic studies of both siblings were obtained and revealed mutations in LPIN2 gene by means of a homozygous single-base pair change in the donor splice site of exon 17 (c. $2327+1 G>C$ ). Both patients underwent different modalities of treatment for MS which involved immunesuppressive and biologic therapies. We observed a significant clinical response to biologic anti-interleukin-1 (IL-1) therapy in our patients. This impressive clinical response indicates the pivotal role of IL-1 in MS pathogenesis. There are limited data on the use of anti-IL-1 therapy in treating MS due to the rarity of the condition. Anti-IL-1 therapy should be considered as a promising treatment for this disease.

\section{G320(P) ADENOSINE DEAMINASE 2 DEFICIENCY WITH A NOVEL VARIANT OF CECR1 GENE MUTATION: RESPONDING TO TUMOR NECROSIS FACTOR ANTAGONIST THERAPY}

B Almoosawi, Z Almusawi, ${ }^{3}$ Hiba Omar, ${ }^{4}$ B Maryam. 'Liverpool Women's Hospital, NHS Foundation Trust, Liverpool, UK; ${ }^{2}$ Salmaniya Medical Complex, Ministry of Health, Bahrain; ${ }^{3}$ King Hamad University Hospital, Bahrain; ${ }^{4}$ Genetics Department, Salmaniya Medical Complex, Ministry of Health, Bahrain

10.1136/archdischild-2020-rcpch.276

Deficiency of Adenosine deaminase 2 (DADA2) syndrome is a chronic, systemic, and inflammatory disorder, characterized by early-onset recurrent strokes, fever, livedo reticularis, and immunodeficiency. We report the case of a 4 -year-old child, a product of consanguineous marriage, who presented with three episodes of hemiparesis within 1 year. She also manifested skin discoloration in the form of livedo reticularis. Workup with magnetic resonance imaging (MRI) of the brain revealed acute infarction in the right aspect of the cerebral peduncle and chronic lacunars infarct in the right thalamus with diffusion restriction. Repeated MRI after 5 months revealed diffuse loss of brain volume. The blood workup showed high inflammatory markers and significantly low adenosine deaminase 2 (ADA2) level. After being on corticosteroid and anticoagulant treatments, she suffered from a recurrent episode of cerebral infarction, after which she was commenced on tumor necrosis factor (TNF)-antagonist therapy in addition to monthly fresh plasma infusion. Thereafter, there was no cerebral insult reported for $>18$ months. The genetic study of the child and her parents revealed a homozygous mutation c. $336 \mathrm{C}>\mathrm{A}$, p. (His112Gln) in the CECR1 gene, and her parents were heterozygous for the same variant. This variant was not previously reported in literature. We would suggest linking this novel variant c. 336C>A, p. (His112Gln) of CECR1 gene mutation with the clinical picture, along with the positive response to TNF-antagonist therapy in the era of ADA2 deficiency syndrome.

\section{British association for paediatric nephrology}

\section{G321 INPATIENT ADMISSION AND ED REATTENDANCE RATES IN A COHORT OF CHILDREN WITH IGA VASCULITIS}

${ }^{1} \mathrm{G}$ Haigh, ${ }^{1,2} \mathrm{~L}$ Oni, ${ }^{3} \mathrm{~S}$ Mulvaney. ${ }^{1}$ Department of Paediatric Nephrology, Alder Hey Children's NHS FT, Liverpool, UK; '2Department of Women's and Children's Health, University of Liverpool, Liverpool, UK; ${ }^{3}$ Department of Emergency Medicine, Alder Hey Children's NHS FT, Liverpool, UK

\subsection{6/archdischild-2020-rcpch.277}

Aim To determine the impact of IgA vasculitis (Henoch Schonlein purpura) on the emergency and acute medical services in a regional paediatric teaching hospital.

Method The case notes of all patients attending Alder Hey Children's Hospital emergency department (ED) and coded as HSP between 1st January 2018 and 31st December 2018 were reviewed retrospectively. Data was collected to establish reasons for admission and reattendance, time between diagnosis and reattendance, and length of inpatient stay on admission.

Results A cohort of 53 patients presented with a new diagnosis of HSP during 2018. 62\% were male, 38\% female. The mean age was 6 years (range 1 to 13 years). 11 patients (21\%) were admitted for symptom management or further investigation. Of these, $4(36 \%)$ were admitted on presentation and $7(64 \%)$ on reattendance. Reasons for admission on presentation included manual blood pressure monitoring (2) and joint pain requiring analgesia (2). All patients were discharged within 24 hours. 14 patients (26\%) reattended ED with problems relating to HSP. Time between diagnosis and reattendance ranged from 1 day to 123 days $($ mean $=9$ days). The primary reasons for reattendance were pain (10/ $14 ; 71 \%)$, worsening/recurring rash $(3 / 14 ; 21 \%)$, and limb swelling $(1 / 14 ; 7 \%)$. Of the 10 patients representing with pain 5 complained of joint pain, 2 of abdominal pain, and 3 of both joint and abdominal pain. $50 \%$ of the reattending patients required admission (joint pain $5 / 7 ; 71 \%$, abdominal pain $2 / 7$; 29\%). All patients requiring admission after reattending with joint pain were admitted for less than 24 hours. The 2 patients with abdominal pain alone were diagnosed with intussusception which resolved with non-operative management and had an inpatient stay of 1 and 3 days respectively.

Conclusion The impact of IgA vasculitis in children extends beyond the potential renal involvement. Patient's experience of pain is a significant factor and leads to a high reattendance/ admission to hospital rate. Ways to improve supporting patients to recover at home are being investigated as specialist intervention was rarely needed. 